\title{
Effect of Er:YAG Laser and Reduced Time of Acid Etching on Bond Strength of Self-adhesive Resin Cement to MTA and Biodentine
}

\author{
Paria Dehghanian $^{1(}$, Fereshteh Shafiei $^{{ }^{*}{ }^{\circledR}}$, Nasrin Kianimanesh $^{2}{ }^{\circledR}$ \\ ${ }^{1}$ Oral and Dental Disease Research Center, Department of Operative Dentistry, School of Dentistry, Shiraz \\ University of Medical Sciences, Shiraz, Iran \\ 2Private Practice, Fellowship in Laser in Dentistry, Vancouver, Canada
}

\section{*Correspondence to Fereshteh Shafiei, \\ Oral and Dental Disease Research Center, Department of Operative Dentistry, School of Dentistry, Shiraz University of Medical Sciences, Shiraz, Iran. Tel: +98-711-36263193; Fax: +98-711-36270325 Email: shafief@sums.ac.ir}

Received: December 19, 2020 Accepted: March 2, 2021

Published online July 18, 2021

\begin{abstract}
Introduction: Considering the recent trend to use mineral trioxide aggregate (MTA) and Biodentine and resin cements, more conservative approaches concurrent with adequate bond strength have always been requested. The present study aimed to evaluate the effect of pretreatment with Er:YAG laser etching versus acid-etching for 5 and 15 seconds on the micro shear bond strength of selfadhesive resin cement (SRC) to MTA and Biodentine.

Materials and Methods: Forty-eight samples of each cement (MTA and Biodentine) were prepared and distributed into four groups based on surface pretreatment: 1) control, no treatment; 2) Er:YAG laser etching with energy of $60 \mathrm{~mJ}$; 3) 5-second acid-etching; 4) 15-second acid-etching. All specimens were cemented using SRC. Microshear bond strengths were tested following 24-hour water storage. Debonded specimens were examined and surface topography was assessed using an atomic force machine (AFM). Data analysis was performed using the two-way ANOVA and Tukey multiple comparisons test.

Results: The three testing groups of laser etch and 5-s and 15-s acid-etch demonstrated a significantly higher SBS than the control group $(P<0.05)$ with negligible differences among them $(P>0.05)$. Furthermore, Biodentine showed better adhesive bonding than MTA in all groups.

Conclusion: Laser etching of $60 \mathrm{~mJ}$ and 5-s acid-etching were as beneficial as 15-s acid-etching in terms of bond strength of SRC to MTA and Bodentine.

Keywords: Acid-etching, Bond strength, Biodentine, Lasers, Mineral trioxide aggregate Angelus, Self-adhesive resin cement
\end{abstract}

\section{Introduction}

Mineral trioxide aggregate (MTA) has been approved as a gold standard in vital pulp therapies due to its ideal performance in terms of biocompatibility, bioactivity, adequate seal, setting ability in the presence of moisture and its resistance to dislodgement. ${ }^{1}$ On the other hand, Biodentine as another novel calcium silicate-based cement (CSC) was subsequently introduced, presenting several more favorable physical properties such as higher compressive strength, lower porosity and accelerated setting reaction. ${ }^{2}$

In most coronal pulpotomy-treated teeth, large restorations are needed. ${ }^{3}$ Indirect approaches are preferred due to improved physical/mechanical properties and simple production of accurate proximal contacts and contours. ${ }^{4} \mathrm{CAD}$-CAM inlay/onlay restorations performed in one session without any need for a temporary phase minimizes leakage and produces an immediate coronal seal. ${ }^{5,6}$
Besides the importance of appropriate vital pulp treatment materials, the outcome of coronal pulpotomy treatment is correlated with the hermetic seal not only at the dentin-CSC interface but also between the restoration and CSC. ${ }^{6}$ In order to obtain this favorable bond in indirect restorations, using resin cements is highly advantageous. The introduction of self-adhesive resin cements (SRCs) to the dental market was a major development in adhesive dentistry with a combination of ideal mechanical and bonding capability characteristics. They have simplified the process of cementation by providing a less technique-sensitive and time-consuming procedure. The acidic monomer in their composition could partially lead to micromechanical retention, and it lacks the necessity of further etching/priming. Nevertheless, in spite of available contrary opinions, ${ }^{8,9}$ some researchers believe that pretreatment with acid prior to self-adhesive luting results in a stronger bond to tooth structures. ${ }^{10-12}$ It might also be constructive for the bond 
of SRCs to CSCs. Bearing in mind the reported adverse effects of acid-etching for 15 seconds on the compressive strength and mechanical properties of CSCs, ${ }^{13}$ reduction in etching time or conservative pretreatment approaches such as lasers could be practical options. In our recent investigations, shortening of acid etching time to 5 seconds has resulted in enhanced resin bond with a universal adhesive to CSCs, preventing the detrimental effect of 15-second phosphoric acid on the compressive strength of MTA and Biodentine. ${ }^{13}$ Also, in the presence of universal adhesive, the Er:YAG laser has appeared to be able to develop satisfactory resin bonding as strong as using 15-s acid etching without compromising the strength of CSCs. ${ }^{14}$ The bond strength between SRC and laser-treated dentin in post space has been examined. ${ }^{15}$ However, little information is available about the effect of laser pretreatment and acid etching when SRCs are bonded to CSCs. Therefore, an investigation into the effect of Er:YAG laser etching versus reduced acid etching time on microshear bond strength of SRC to MTA and Biodentine was the main goal in the current study.

The supposition that different surface pretreatments would not affect the microshear bond strength of MTA and Biodentine to SRC could be assumed as the null hypothesis of the present examination.

\section{Materials and Methods}

Ninety-six cylindrical acrylic specimens with a central hole of $2 \mathrm{~mm}$ in diameter and height were prepared and filled with two groups of either MTA (MTA, Angelus, Londrina, Brazil, Lot \# 40422) or Biodentine (BD, Septodont, France, lot \# B19471) following the manufacturer's recommendations. Wet cotton pellets covered the blocks and subsequently, the samples were stored in an incubator at $37^{\circ} \mathrm{C}$ under about $100 \%$ humidity for 72 hours to reach their final setting. Afterwards, with the purpose of obtaining a uniform surface, the specimens were polished by means of 600-grit silicon carbide paper for $1 \mathrm{~min}$ and distributed into four groups $(n=12)$ based on the surface treatment: group 1) No surface conditioning (control); groups 2 and 3) 37\% phosphoric acid for 5 and $15 \mathrm{~s}$, respectively; group 4) Er:YAG laser irradiation. In the last group, the Er:YAG laser (LightWalker ATS, Fotona, Slovenia) with $60 \mathrm{~mJ}$ energy $\left(11.94 \mathrm{~J} / \mathrm{cm}^{2}\right)$ was applied uniformly and perpendicularly to the surfaces in a noncontact mode at a distance of 1-2 mm for 10 seconds. The procedure was performed using a $0.8 \mathrm{~mm}$ diameter tip of the 'H14' handpiece with the following parameters: 2940 $\mathrm{nm}, 10 \mathrm{~Hz}, \mathrm{MSP}$ mode (100 $\mu \mathrm{s})$, water 8, and air 4 .

Following surface treatment, cylindrical elastic molds $(1 \times 1 \mathrm{~mm})$ were placed on the specimens and filled with SRC (Kuraray Noritake Dental Inc, Sakazu, Kurashiki, Okayama, Japan, Lot \# 033BBA). Using a light-curing unit (VIP Junior, Bisco), they were light-cured at a light intensity of $600 \mathrm{~mW} / \mathrm{cm}^{2}$ for 20 seconds. The samples were then stored in distilled water for 24 hours at $37^{\circ} \mathrm{C}$. Bond strengths in microshear ( $\mu \mathrm{SBS}$ ) were tested in $\mathrm{MPa}$, using a universal testing machine (Zwick, Ulm, Germany) at a crosshead speed of $1 \mathrm{~mm} / \mathrm{min}$. Subsequently, the debonded surfaces were evaluated under a stereomicroscope at $\times 20$ magnification to categorize the failure modes as follows: (1) adhesive failure between CSC and resin cement; (2) cohesive failure within the resin cement or in the bulk of CSC; and (3) mixed failure, a combination of adhesive and cohesive failure modes.

Two further specimens from each group of both CSCs were prepared with the same method as mentioned previously. In order to observe topographical features, the sample surfaces were scanned with an atomic force machine (AFM) (Naio AFM, NanoSurf, Switzerland) via a sharp silicon cantilever (Tap150Al-G, Budget Sensor, Bulgaria) in a dynamic non-contact mode, with the resonance frequency of $160 \mathrm{kHz}$ and stiffness constant of $0.2 \mathrm{~N} / \mathrm{m}$.

\section{Statistical Analysis}

The data were submitted to two-way ANOVA analysis and the Tukey test at a significance level of 5\%. All statistical analyses were performed by employing SPSS, version 16.0 software (SPSS Inc, Chicago, IL, USA).

\section{Results}

The comparison of the mean SBS and contributing standard deviations of the four groups in each CSC are presented in Table1. According to the two-way ANOVA, the effects of CSC type and treatment were significant $(P=0.01, P<0.001)$; no interaction between CSCs and pretreatments was found. This finding declares that both CSCs demonstrate a similar trend in their reaction to pretreatments. Thus, the data were paraphrased and compared using Tukey post hoc tests. Accordingly, variation in the type of CSC led to a significant effect on the $\mu$ SBS of SRC to the underneath pulp capping material $(P=0.01)$ in such a way that Biodentine showed a better performance. In addition, although the three testing groups of laser etch and acid etch for 5 and 15 seconds yielded a significantly higher $\mu \mathrm{SBS}$ than the control group $(P<0.05)$, no differences were observed among them $(P$ $>0.05)$.

In the control group of MTA and Biodentine, all failures were adhesive, while in all treated groups of MTA and Biodentine, the adhesive failure was the main failure with a few mixed failures observed in some specimens.

The representative $3 \mathrm{D}$ images and micrographs of atomic force microscopy related to the topography of MTA and BD in all groups are illustrated in Figures 1 and 2 respectively. The samples were entirely studied at the same magnification $(10 \times 10 \mu \mathrm{m})$. Bright and dark areas indicate height and depth respectively. AFM analysis showed a fairly even and uniform surface in the 
Table 1. Means and Standard Deviations of Microshear Bond Strengths (MPa) of Self-adhesive Resin Cement to MTA and Biodentine Affected by Different Etching Modes

\begin{tabular}{|c|c|c|c|c|}
\hline \multirow{2}{*}{$\begin{array}{l}\text { CSC } \\
\text { Etching mode }\end{array}$} & \multicolumn{2}{|c|}{ MTA } & \multicolumn{2}{|c|}{ Biodentine } \\
\hline & Mean $(\mathrm{SD})^{*}$ & Failure Mode & Mean (SD) & Failure Mode \\
\hline Self-adhesive & $3.28(1.00)^{\mathrm{Aa}}$ & $12 / 0 / 0$ & $4.06(1.46)^{\mathrm{Ab}}$ & $12 / 0 / 0$ \\
\hline Acid etch $5 \mathrm{~s}$ & $5.9(1.50)^{\mathrm{Ba}}$ & $8 / 2 / 2$ & $6.58(1.64)^{\mathrm{Bb}}$ & $10 / 2 / 0$ \\
\hline Acid etch $15 \mathrm{~s}$ & $5.84(1.48)^{\mathrm{Ba}}$ & $9 / 1 / 2$ & $6.7(1.30)^{\mathrm{Bb}}$ & $8 / 3 / 1$ \\
\hline Laser etch 60 & $5.74(1.50)^{\mathrm{Ba}}$ & $10 / 1 / 1$ & $6.54(1.52)^{\mathrm{Bb}}$ & $11 / 1 / 0$ \\
\hline$P$ value & \multicolumn{2}{|c|}{$<0.05$} & \multicolumn{2}{|c|}{$<0.05$} \\
\hline
\end{tabular}

The numbers in the failure mode column represent adhesive failure, cohesive failure within CSC or resin cement, and mixed failure respectively.

*Different superscript capital letters in each column and lowercase letters in each row indicate a statistically significant difference $(P<0.05)$.

control groups of both CSCs. Almost all etching methods modified the surface topography by raising the roughness; in 15 seconds acid etch and laser etch groups for both MTA and Biodentine, however, this alteration was more obvious, providing a porous pattern through increased height/depth of the peaks/valleys.

\section{Discussion}

The present study investigated the impact of laser etching or 5-s acid etching versus 15-s acid etching (as a practical standard etching time) of CSCs on the bonding ability of an SRC. Our previous findings indicated that 5 -second etching and $60 \mathrm{~mJ}$ laser irradiation had no detrimental effect on the strength of CSCs contrary to 15-second etching time. ${ }^{13,14}$ Therefore, these pretreatments were applied in the current study; consequently, they were as effective as 15-second etching in the promotion of SRC bonding to both CSCs. This result was in agreement with our recent studies with universal adhesive in the case of MTA, but not for Biodentine. The higher viscosity of SRC compared to that of universal adhesive could justify this difference in the obtained results.

The parameters of Er:YAG laser irradiation used in the current study were based on the outcome of a recent study demonstrating $60 \mathrm{~mJ}$ laser etching as the optimal energy for the creation of a retentive pattern without a destructive impact on the strength of CSCs. ${ }^{14}$ Compared to the control group, both the mentioned laser etching mode and 5-s acid etching were successful in improving the bond strength of SRC to CSCs.

The presence of some acidic monomers in SRCs was demonstrated to interact with the calcium ions. ${ }^{16}$ MTA and Biodentine cements produce a high amount of calcium ions from calcium hydroxide, a by-product of hydration, and from the decomposition of calcium silicate hydrate. ${ }^{10,17} \mathrm{~A}$ chemical bond is established as a result of a chelation process in which functional monomers such as 10-methacryloyloxydecyl dihydrogen phosphate (10-MDP) react with calcium ions, increasing the bond strength. ${ }^{2,18}$ Nevertheless, increased surface energy and wettability of CSCs following acid or laser etching were more vital. This may provide intimate contact of self-adhesive cement on the surface of CSC, facilitating chemical bonding. ${ }^{19}$

AFM observations were in line with obtained results. The porous pattern with multiple heights and depths provided by all treatments could justify the increased bonding to CSCs. It is noteworthy that 15-s acid etch and laser etch groups in both MTA and Biodentine materials altered the surface more considerably. Furthermore, according to our previous SEM observations, these treatments could create micro-retentive patterns on the surface of CSC that might be responsible for higher resin bonding to MTA and Biodentine. The higher bond strength of SRC to MTA and glass ionomer as root perforation materials compared to Portland cement has been attributed to their surface roughness, especially for GI. ${ }^{18}$ However, those authors reported that $\mu \mathrm{SBS}$ values obtained in their study were lower than those of value range required to prevent gap formation at the cement interface. Contrarily, laser and acid etching seem to achieve this level of bond strength.

In order to simulate a clinical environment, MTA and Biodentine should be stored in a humid environment for about 72 hours to complete their ultimate setting. ${ }^{20}$ While a 3D scan is carried out in the second session as a computerized impression, the pretreatment conditioning could be performed prior to the permanent cementation of final indirect restoration with SRC in the same session; therefore, the bonding of SRC could be carried out after 72 hours without any delay for the fabrication of indirect restoration.

Biodentine demonstrated higher microshear bond strength compared to MTA, regardless of the etching method. This outcome was in line with the available data reported previously $y^{13,14,21}$ and this could be as a result of high density and the superficial porosity of Biodentine providing micromechanical bonding. Hypothetically, the 10-MDP monomer may bind chemically to the calcium in Biodentine and promote chemical adhesion and micromechanical attachment. ${ }^{21,22}$

Present results cannot be directly applied to the clinical situation without taking into account the limitations of 

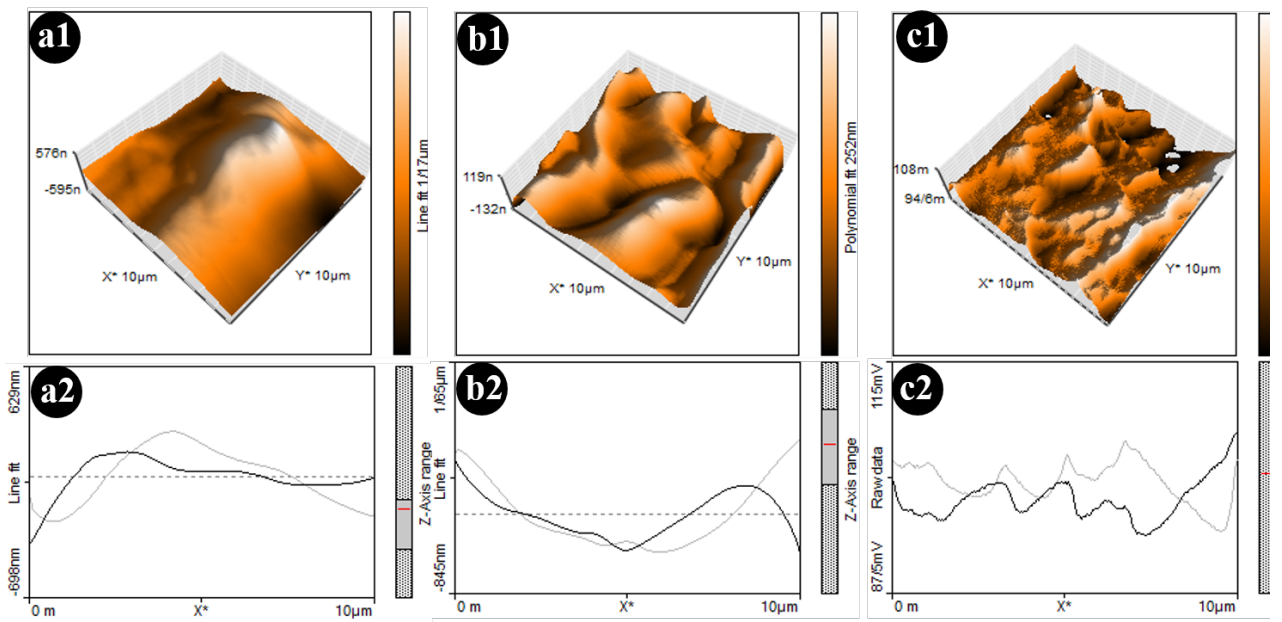

Figure 1. AFM Images of MTA in Two Views of 3D Images (a1-d1) and Topographical Micrographs (a2-d2). Bright and dark areas indicate height and depth respectively. a1 and a2) non-etch surface, relatively flat and uniform surface. b1 and b2) $5 \mathrm{~s}$ acid etch surface, more irregularities. $\mathrm{c} 1$ and c2) $15 \mathrm{~s}$ acid etch surface, micro retentive appearance as a number of concavities and convexities. $\mathrm{d} 1 \mathrm{and} \mathrm{d} 2$ ) laser etch, valley-like appearance full of peaks and valleys.
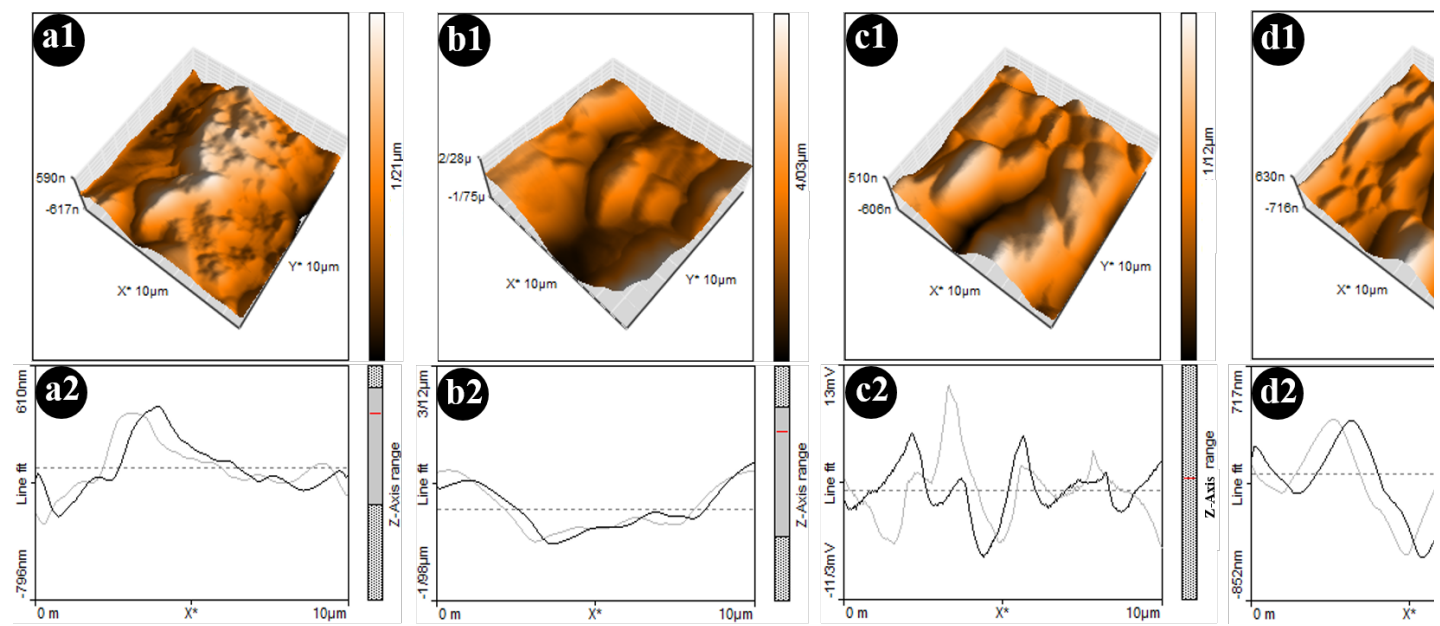

Figure 2. AFM Images of Biodentine in Two Views of 3D Images (a1-d1) and topographical Micrographs (a2-d2). Bright and dark areas indicate height and depth respectively. a1 and a2) non-etch surface, relatively flat and uniform surface. b1 and b2) 5s acid etch surface. c1 and c2) 15s acid-etch surface, micro-retentive appearance as a number of concavities and convexities. d1 and d2) laser etch, more irregularities.

this study. In clinical conditions, the thinner thickness of cement layers would be applied in a cavity with higher $\mathrm{C}$-factor compared to the flat surface used for in vitro studies. Moreover, different types of stresses in oral environment like $\mathrm{pH}$ changes, thermal-cycling, occlusal loads and challenges due to the presence of enzymes could damage the bonding effectiveness of the luting agents. Future clinical trials are necessary to validate the outcomes of the present study.

\section{Conclusion}

Considering the limitations of this study, it could be deduced that the surface pretreatment of MTA and Biodentine with both laser and acid etching is more advantageous than no pretreatment in terms of their bond strength to SRC.

Since there was no difference between $15 \mathrm{~s}$ and $5 \mathrm{~s}$ acid etching and laser etching, treatment with either laser or $5 \mathrm{~s}$ phosphoric acid could be suggested due to their lower destructive effects.

\section{Ethical Considerations}

This article does not contain any studies with human participants or animals performed by any of the authors.

\section{Conflict of Interests}

The authors declare that they have no conflict of interest.

\section{Funding}


This work was supported by Shiraz University of Medical Sciences, Shiraz, Iran.

\section{Acknowledgment}

The authors thank the vice-chancellery for research, Shiraz University of Medical Sciences, Shiraz, Iran, for supporting the current research (Grant \# 17362).

\section{References}

1. Kang CM, Sun Y, Song JS, Pang NS, Roh BD, Lee CY, et al. A randomized controlled trial of various MTA materials for partial pulpotomy in permanent teeth. J Dent. 2017;60:813. doi: 10.1016/j.jdent.2016.07.015

2. Rajasekharan S, Martens LC, Cauwels RG, Verbeeck RM. Biodentine material characteristics and clinical applications: a review of the literature. Eur Arch Paediatr Dent. 2014;15(3):147-158. doi: 10.1007/s40368-014-0114-3

3. Taha N, Ahmad M, Ghanim A. Assessment of mineral trioxide aggregate pulpotomy in mature permanent teeth with carious exposures. Int Endod J. 2017;50(2):117-125. doi: 10.1111/iej.12605

4. Spitznagel FA, Horvath SD, Guess PC, Blatz MB. Resin bond to indirect composite and new ceramic/polymer materials: a review of the literature. J Esthet Restor Dent. 2014;26(6):382-393. doi: 10.1111/jerd.12100

5. Anadioti E, Aquilino SA, Gratton DG, Holloway JA, Denry IL, Thomas GW, Qian F. Internal fit of pressed and computer-aided design/computer-aided manufacturing ceramic crowns made from digital and conventional impressions. J Prosthet Dent. 2015;113(4):304-309. doi: 10.1016/j.prosdent.2014.09.015

6. Tabassum S, Khan FR. Failure of endodontic treatment: The usual suspects. Eur J Dent. 2016;10(1):144-147. doi: 10.4103/1305-7456.175682

7. Manso AP, Carvalho RM. Dental Cements for Luting and Bonding Restorations: Self-Adhesive Resin Cements. Dent Clin North Am. 2017;61(4):821-834. doi: 10.1016/j. cden.2017.06.006

8. Mushashe AM, Gonzaga CC, Cunha LF, Furuse AY, Moro A, Correr GM. Effect of Enamel and Dentin Surface Treatment on the Self-Adhesive Resin Cement Bond Strength. Braz Dent J. 2016;27(5):537-542. doi: 10.1590/0103-6440201600445

9. Rodrigues RF, Ramos CM, Francisconi PA, Borges AFS. The shear bond strength of self-adhesive resin cements to dentin and enamel: an in vitro study. J Prosthet Dent. 2015;113(3):220-227. doi: 10.1016/j.prosdent.2014.08.008

10. Pisani-Proenca J, Erhardt MC, Amaral R, Valandro LF, Bottino MA, Del Castillo-Salmeron R. Influence of different surface conditioning protocols on microtensile bond strength of self-adhesive resin cements to dentin. $J$ Prosthet Dent. 2011;105(4):227-235. doi: 10.1016/S00223913(11)60037-1

11. Pavan S, dos Santos PH, Berger S, Bedran-Russo AK. The effect of dentin pretreatment on the microtensile bond strength of self-adhesive resin cements. $J$ Prosthet Dent. 2010;104(4):258-264. doi: 10.1016/S0022-3913(10)60134-5

12. Shafiei F, Jowkar Z, Hosseini N. Influence of Cavity Pretreatments on the Fracture Resistance of Premolars with Self-adhesive Cemented Composite Inlay. J Contemp Dent Pract. 2019;20(7):829. doi: 10.5005/jp-journals-10024-2605

13. Shafiei F, Doozandeh M, Gharibpour F, Adl A. Effect of reducing acid-etching duration time on compressive strength and bonding of a universal adhesive to calcium silicate cements. Int Endod J. 2019;52(4):530-539. doi: 10.1111/iej.13026

14. Shafiei F, Dehghanian P, Memarpour M, Sabeti M. Effect of Er: YAG laser etching on topography, microstructure, compressive strength and bond strength of a universal adhesive to calcium silicate cements. Clin oral investig. 2020;24(2):711-718. doi: 10.1007/s00784-019-02903-2

15. Simundic Munitic M, Bago I, Glockner K, Kqiku L, Gabric D, Anic I. Effect of Different Laser Treatments on the Bond Strength of Intracanal Fiber Posts Cemented with a Self-Adhesive Resin Cement. J Prosthodont. 2019 Jan;28(1):e290-e296. doi: 10.1111/jopr.12738

16. Nagakane K, Yoshida Y, Hirata I, Fukuda R, Nakayama Y, Shirai K, et al. Analysis of chemical interaction of 4-MET with hydroxyapatite using XPS. Dent Mater J. 2006;25(4):645-649. doi: 10.4012/dmj.25.645

17. Camilleri J. Scanning electron microscopic evaluation of the material interface of adjacent layers of dental materials. Dent Mater. 2011;27(9):870-878. doi: 10.1016/j. dental.2011.04.013

18. Lemos Martins Sicuro S, Gabardo MC, Castiglia Gonzaga C, Dias Morais N, Baratto-Filho F, Correr Nolasco GM, et al. Bond Strength of Self-adhesive Resin Cement to Different Root Perforation Materials. J Endod. 2016;42(12):18191821. doi: 10.1016/j.joen.2016.08.019

19. Lee S-R, Moon Y-M, Hwang S-J, Seo M-S. The effect of acid-etch procedure on the bond between composite resin and mineral trioxide aggregate. Korean J Dent Mater. 2014;41(1):53-57. doi: 10.14815/kjdm.2014.41.1.53

20. Bodanezi A, Carvalho N, Silva D, Bernardineli N, Bramante CM, Garcia RB, Moraes IGd. Immediate and delayed solubility of mineral trioxide aggregate and Portland cement. J Appl Oral Sci. 2008;16(2):127-131. doi: 10.1590/ s1678-77572008000200009

21. Hashem DF, Foxton R, Manoharan A, Watson TF, Banerjee A. The physical characteristics of resin composite-calcium silicate interface as part of a layered/laminate adhesive restoration. Dent Mater. 2014;30(3):343-349. doi: 10.1016/j. dental.2013.12.010

22. Yoshida Y, Nagakane K, Fukuda R, Nakayama Y, Okazaki $\mathrm{M}$, Shintani $\mathrm{H}$,et al. Comparative study on adhesive performance of functional monomers. J Dent Res. 2004;83(6):454-458. doi: 10.1177/154405910408300604 